Characterization of the Medipix3 pixel readout chip

This article has been downloaded from IOPscience. Please scroll down to see the full text article.

2011 JINST 6 C01052

(http://iopscience.iop.org/1748-0221/6/01/C01052)

View the table of contents for this issue, or go to the journal homepage for more

Download details:

IP Address: 137.138.124.233

The article was downloaded on 27/07/2012 at 08:32

Please note that terms and conditions apply. 
$12^{\text {th }}$ INTERNATIONAL WORKSHOP ON RADIATION IMAGING DETECTORS,

JULY $11^{\text {th }}-15^{\text {th }} 2010$,

RoBinson College, CAMBRIDGE U.K.

\title{
Characterization of the Medipix3 pixel readout chip
}

\author{
R. Ballabriga, ${ }^{a}{ }^{1}$ G. Blaj, ${ }^{a}$ M. Campbell, ${ }^{a}$ M. Fiederle,${ }^{b}$ D. Greiffenberg, ${ }^{b}$ \\ E.H.M. Heijne, ${ }^{a}$ X. Llopart, ${ }^{a}$ R. Plackett, ${ }^{a, c}$ S. Procz, ${ }^{b}$ L. Tlustos, ${ }^{a}$ D. Turecek ${ }^{d}$ \\ and W. Wong ${ }^{a}$
}

${ }^{a}$ CERN, PH Department, 1211 Geneva 23, Switzerland

${ }^{b}$ Freiburger Materialforschugszentrum, FMF, Stefan-meier-Straße 21, D-7910 Freiburg, Germany

${ }^{c}$ Department of Physics and Astronomy, University of Glasgow, Kelvin Building, University Avenue, Glasgow, G12 8QQ Glasgow, Scotland, U.K.

${ }^{d}$ Institute of Experimental and Applied Physics, Czech Technical University, Horská 3a/22, 12800 Prague 2, Prague, Czech Republic

E-mail: Rafael.Ballabriga@cern.ch

ABSTRACT: The Medipix3 chip is a hybrid pixel detector readout chip working in Single Photon Counting Mode. It has been developed with a new front-end architecture aimed at eliminating the spectral distortion produced by charge diffusion in highly segmented semiconductor detectors. In the new architecture charge deposited in overlapping clusters of four pixels is summed event-byevent and the incoming quantum is assigned as a single hit to the summing circuit with the biggest charge deposit (this mode of operation is called Charge Summing Mode (CSM)). In Single Pixel Mode (SPM) the charge reconstruction and the communication between neighbouring pixels is disabled. This is the operating mode in traditional detector systems.

This paper presents the results of the characterization of the chip with electrical stimuli and radioactive sources.

KEYWORDS: Front-end electronics for detector readout; Analogue electronic circuits; Digital electronic circuits

\footnotetext{
${ }^{1}$ Corresponding author.
} 


\section{Contents}

1 Introduction 1

2 Brief description of the chip architecture 2

3 Calibration using X-ray fluorescent targets 3

4 Count rate behavior $\quad 4$

5 Imaging 5

6 Summary and further work $\quad 8$

\section{Introduction}

This paper reports on measurements using the first version of the Medipix 3 readout chip connected to a $\mathrm{Si}$ sensor.

Single photon counting pixel detectors have great potential for X-ray imaging and other particle counting applications. Individual X-ray photons are detected and discriminated cleanly from noise using pixel pulse processing electronics which is connected via bump bonding to an equally segmented semiconductor detector. The number of incoming quanta are then counted in a local on-pixel register. Moreover, multiple thresholds may be applied to bin the incoming X-ray photons according to their energy and if one counting register is available per threshold a spectroscopic image can be produced. However, in some cases the charge deposition in the sensor is spread over a large volume. Charge diffusion during carrier drift and collection may lead to charge sharing between neighbouring pixels. Charge diffusion is an important factor when the sensor thickness exceeds the pitch of the pixels. Fluorescence can also be an important factor when high $\mathrm{Z}$ detector materials are used and the mean free path of the emitted photon is in the same range as the pixel pitch.

The Medipix3 pixel detector readout chip [1] seeks to mitigate the effects of charge sharing by first summing the charge detected in neighbouring pixels prior to allocating the hit for counting to the summing node with the highest total charge in the neighbourhood.

In the first section the architecture of the chip and the pixel are reviewed and some of the key electrical parameters highlighted. In order to confirm the accuracy of the electrical measurements a calibration procedure with X-rays generated in fluorescent targets was carried out. This procedure and the results are presented in the second section. A key performance parameter of the detection system is the linearity of on-pixel counting as a function of incoming photon flux. The spectroscopic behaviour of the chip in both single pixel and charge summing modes were also measured. Those measurements are presented in the $3^{\text {rd }}$ section. The imaging performance of the chip was evaluated in single pixel mode and in charge summing mode with Si sensors. Finally we summarize the results and give some indication of the future direction. 


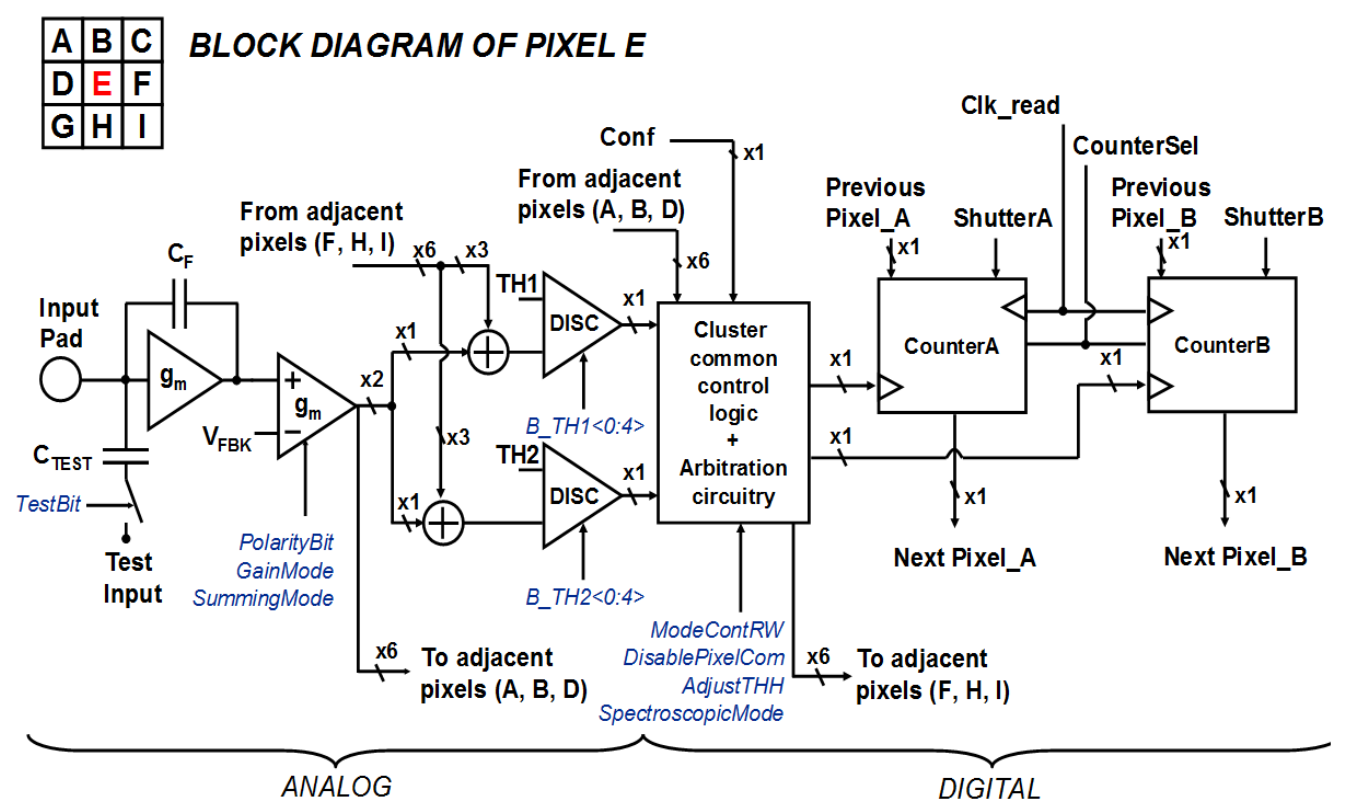

Figure 1. Medipix3 pixel schematic diagram. The input charge is integrated in the preamplifier feedback capacitance $\left(\mathrm{C}_{\mathrm{F}}\right)$. A shaping amplifier processes the preamplifier output generating a number of copies which are connected to summing nodes. Each summing node is associated to a discriminator. The digital part contains pulse processing logic, arbitration circuits for hit assignment, latches for pixel configuration storage and two configurable depth counters.

\section{Brief description of the chip architecture}

The architecture of the chip has been described elsewhere [1-3]. This section summarizes the main features.

The schematic block diagram of the Medipix 3 pixel cell is shown in figure 1. Charge collected in the sensor pixel is integrated on the $14 \mathrm{fF}$ feedback capacitor of the front-end preamplifier. The preamplifier is sensitive to both positive and negative incoming charge and also has a low frequency feedback loop (not shown) which is capable of sinking or sourcing detector leakage current (up to $+10 \mathrm{nA}$ or $5 \mathrm{nA}$ ). The output of the preamplifier is connected to a shaping amplifier which converts this voltage to a number of current copies. The peaking time of the shaper is approximately $120 \mathrm{~ns}$. Two discriminators and two counters are available per pixel. The depth of the counters can be configured as 1-bit, 4-bit or 12-bit. There is also the possibility to configure the counters as a single 24-bit depth counter at the expense of having a single energy threshold per pixel. Furthermore each pixel has a number of configuration bits for enabling or disabling a test pulse through a test injection capacitance, pixel masking, shaper gain selection and 5 bits each for threshold for fine tuning for each discriminator. The pixel occupies an area of $55 \mu \mathrm{m} \times 55 \mu \mathrm{m}$.

In single pixel mode the amplitude of the shaped current from the pixel is compared with a threshold current which is applied globally from a threshold DAC on the periphery. When the amplitude of the signal pulse exceeds the threshold the counter connected to the discriminator output is incremented. In single pixel mode the shaper provides two current copies which are compared to two different thresholds when data taking and readout are sequential (sequential RW). 


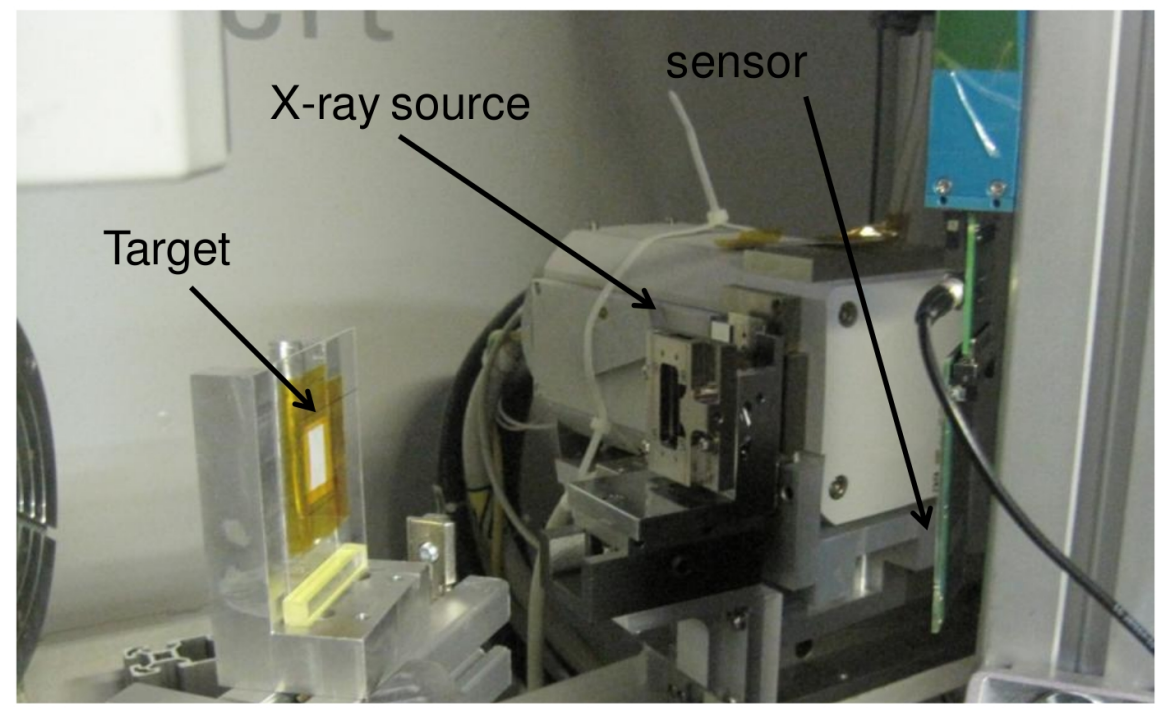

Figure 2. Photo of the test calibration setup showing a Medipix3 Si assembly configured to detect the fluorescence photons generated in the target material when illuminated by a standard laboratory X-ray source.

If dead time free operation is desired then only one threshold is used and one counter is incremented while the other is being read out (continuous RW).

In charge summing mode copies of the shaper current are transmitted to each pixel corner. There the 4 currents from the immediate neighbours are summed prior to the discrimination and charge allocation processes. A hit is only counted provided that a particular corner has a greater charge sum than its neighbours. In practice the hit arbitration is done by comparing the timing properties of the discriminator outputs in the neighbourhood. The summing corner with the highest charge takes the hit and suppresses the neighbouring hits. This ensures that each hit is counted once and only once. As with single pixel mode there are 2 discriminators and counters per pixel corner providing 2 thresholds. In continuous RW only one discriminator per corner is used.

The chip can be further configured such that only one input pad in 4 is connected to a sensor with $110 \mu \mathrm{m}$ pitch. In this case up to 8 discriminators and counters are available per large pixel and charge summing can take place over a total area of $220 \mu \mathrm{m} \times 220 \mu \mathrm{m}$.

The noise of the front-end was measured using a test pulse to be $\sim 75 \mathrm{e} \mathrm{rms} \mathrm{in} \mathrm{single} \mathrm{pixel} \mathrm{mode}$ and $\sim 150$ e rms in charge summing mode. The threshold variation from pixel to pixel in single pixel modes is $140 \mathrm{e}$ rms after threshold tuning, a value which is about three times higher than the design value. The threshold can be varied monotonically from $\sim 950$ e to $\sim 15000 \mathrm{e}$. The power consumption of the chip is $600 \mathrm{~mW}$ in single pixel mode and $900 \mathrm{~mW}$ in charge summing mode.

\section{Calibration using X-ray fluorescent targets}

Measurements were performed on individual chips which have been connected by bump bonding to standard high resistivity $300 \mu \mathrm{m}$ thick silicon pixel sensors forming single chip assemblies. One assembly was mounted in the setup shown in figure 2. Various materials were placed in the position of the target and the chip was mounted at such an angle that it was outside of the broad spectrum 

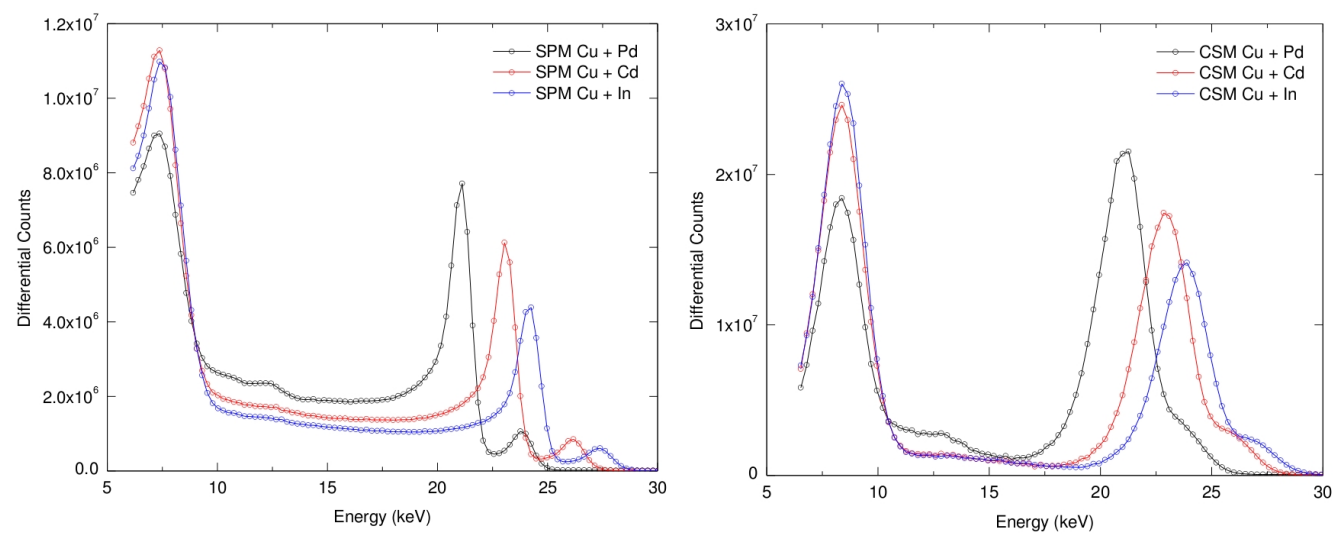

Figure 3. X-ray spectra produced using different target materials (Copper, Palladium, Cadmium and Indium). The spectra seen when the detector is configured in single pixel mode are shown on the left. The spectra measured when the chip is programmed in charge summing mode are shown on the right. The charge sharing tail is greatly reduced in the latter configuration. The pixel-to-pixel offset was corrected in the plotted data.

direct beam and received mostly fluorescence photons from the target material and scattered copper radiation from the tube anode. The global threshold of the chip was then scanned and the number of counts per pixel was recorded. This provides a cumulative spectra of the charge detected by the pixels. The counts were then differentiated with respect to the threshold value providing the spectra observed by the pixels as shown in figure 3 .

It is quite clear by comparing the resultant spectra of the single pixel mode (left) with that of the charge summing mode (right) that the charge summing scheme effectively reduces the charge sharing tail seen in the single pixel mode. Moreover, the peaks of the spectra observed were used to calibrate the threshold current versus input charge, as seen in figure 4.

The noise of the front-end in single pixel mode was measured to be $\sim 75 \mathrm{e} \mathrm{rms}$ and the residual threshold variation after threshold tuning was $\sim 140 \mathrm{e} \mathrm{rms.} \mathrm{In} \mathrm{charge} \mathrm{summing} \mathrm{mode} \mathrm{the} \mathrm{noise} \mathrm{was}$ $\sim 150 \mathrm{e} \mathrm{rms}$ and the threshold variation $\sim 220 \mathrm{e} \mathrm{rms}$. While the noise figures are in good agreement with the simulation the threshold dispersion is significantly worse than expected.

\section{Count rate behavior}

A characteristic of photon counting systems is the requirement of a minimum amount of time between two events in order to be recorded as two separate pulses. This time is called dead time of the counting system and is mainly determined by the speed of the readout electronics [4]. Measurements were made using a laboratory $\mathrm{X}$-ray source placing a $\mathrm{Si}$ assembly as close as possible to the output of the tube and varying the tube current. The target of the tube was $\mathrm{W}$, the tube had a $0.6 \mathrm{~mm} \mathrm{Al} \mathrm{filter} \mathrm{at} \mathrm{the} \mathrm{output} \mathrm{and} \mathrm{the} \mathrm{tube} \mathrm{voltage} \mathrm{was} 40 \mathrm{kV}$. Only $\sim 200$ pixels were used in this measurement as these were exposed to a uniformly high flux in the center of the beam. Figure 5 shows the measured count rate as a function of the interaction rate for the chip in single pixel mode and charge summing mode for three different values of the DAC that controls the return to zero of the preamplifier output voltage. There is a notable difference in the behaviour at higher 

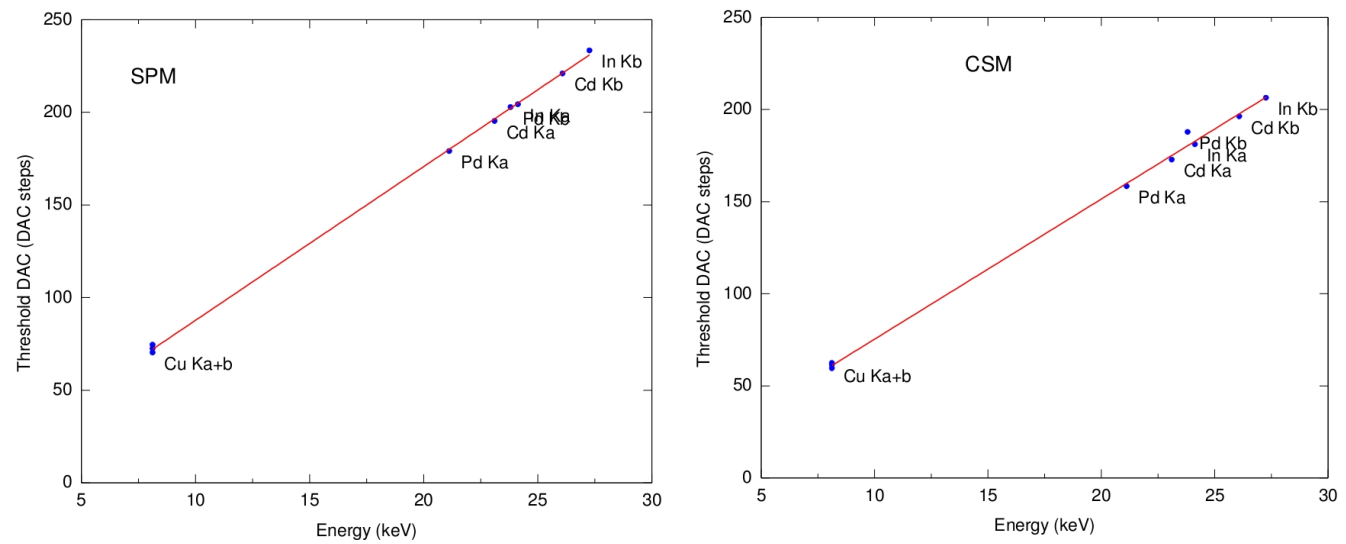

Figure 4. Linearity of the detector with energy after fitting the measured spectra with the energies of the emitted fluorescence photons.
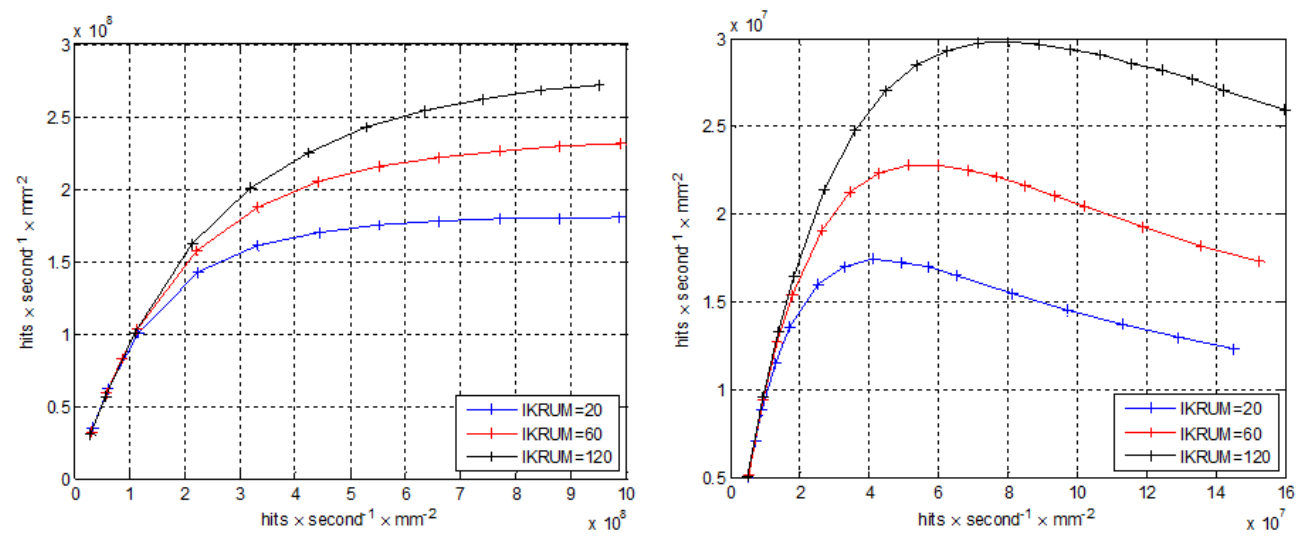

Figure 5. Count rate measurements for the chip in single pixel and charge summing modes for different values of the DAC that controls the return to baseline of the preamplifier output signal.

flux rates. The pixel intercommunication used in the Medipix 3 charge summing scheme has an influence on the system's ability to cope with high fluxes because when a photon is recorded by a pixel its adjacent 8 neighbours become paralyzed from getting the hit. In charge summing mode the chip is able to provide a monotonic behaviour up to $17 \times 10^{6} \mathrm{ph} / \mathrm{mm}^{2} / \mathrm{s}$. In single pixel mode, for the same conditions the flux the system is able to process is a factor approximately 10 times higher. The dead time is $\sim 0.7 \mu \mathrm{s}$ in single pixel mode and $\sim 7 \mu \mathrm{s}$ in charge summing mode.

Increasing the DAC controlling the return to zero of the preamplifier has a beneficial impact in the flux the system is able to process but other parameters of the system e.g. noise and minimum threshold are degraded.

\section{Imaging}

A number of images were taken using the Si assembly. Figure 6 shows data from the low threshold and the high threshold counter taken in the same shot using single pixel mode with a laboratory $\mathrm{X}$-ray source with $\mathrm{W}$ target, $2 \mathrm{~mm} \mathrm{Al}$ filter, tube voltage $50 \mathrm{kV}$ and exposure time $1 \mathrm{~s}$. In spite of the 

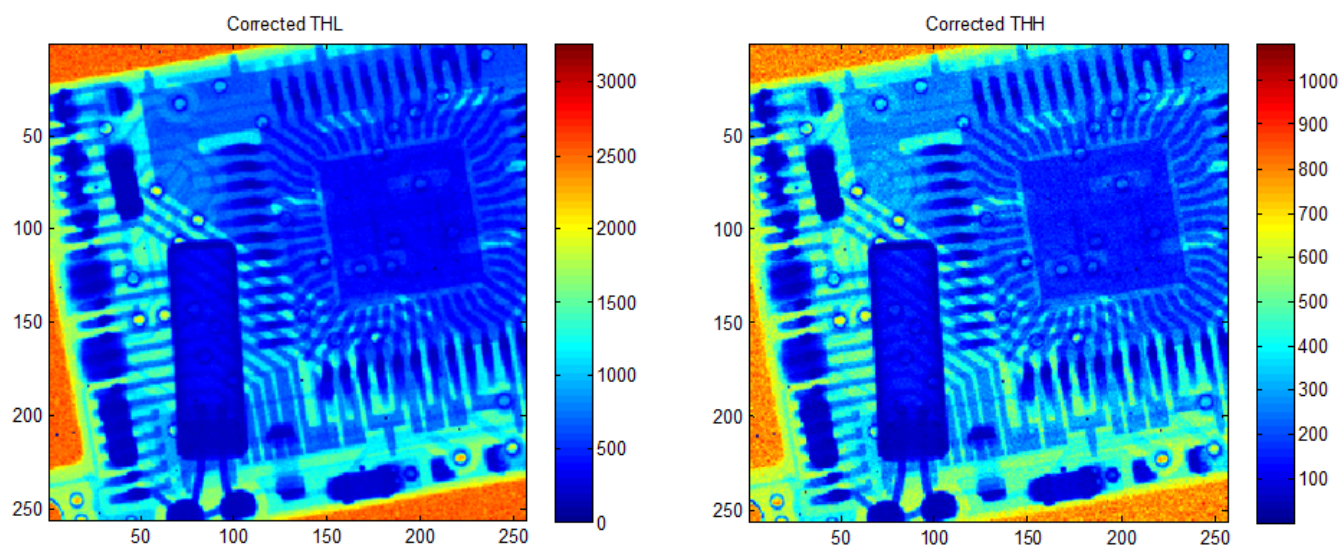

Figure 6. Two X-ray images of an electronic device taken in one shot and in single pixel mode. The left image has a low threshold $\sim 8.5 \mathrm{keV}$ and the right image has a higher threshold of $\sim 34 \mathrm{keV}$.
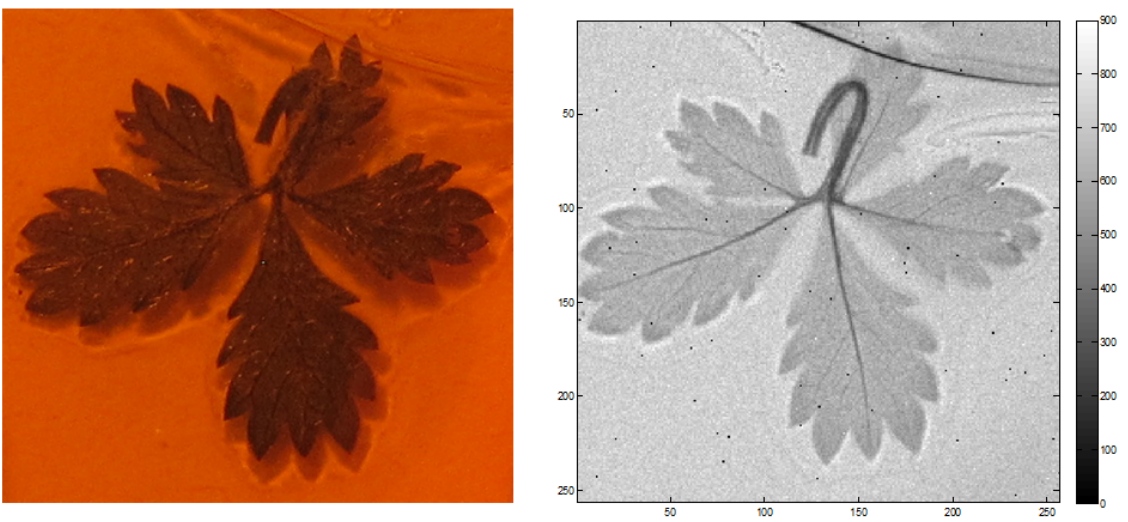

Figure 7. Image of a small leaf. The optical photograph is shown on the left. On the right, the contrast image taken with the Medipix 3 chip using an ${ }^{55} \mathrm{Fe}$ source and an exposure time of 12 hours is shown. The black dots correspond to masked pixels due to their higher threshold offset after execution of the equalization procedure.
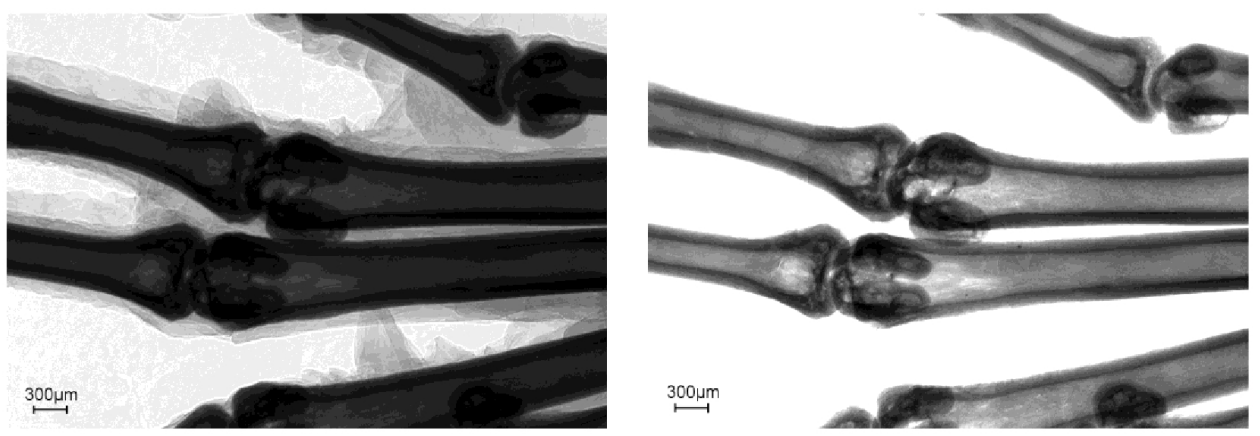

Figure 8. Foot of a mouse paw in 10x magnification (related to detector). The left image uses the full histogram range and provides details for the tissue. The right image has a cropped histogram for high contrast for bones. 


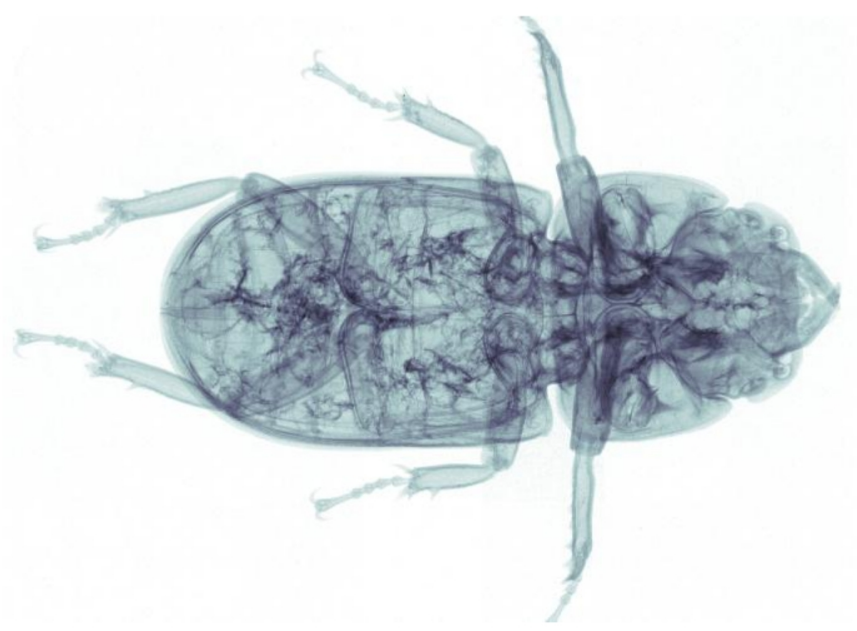

Figure 9. X-ray image of a lesser stag beetle (Dorcus parallelipipedus). All internal parts inside the chitin shell are visible just like outer body parts.
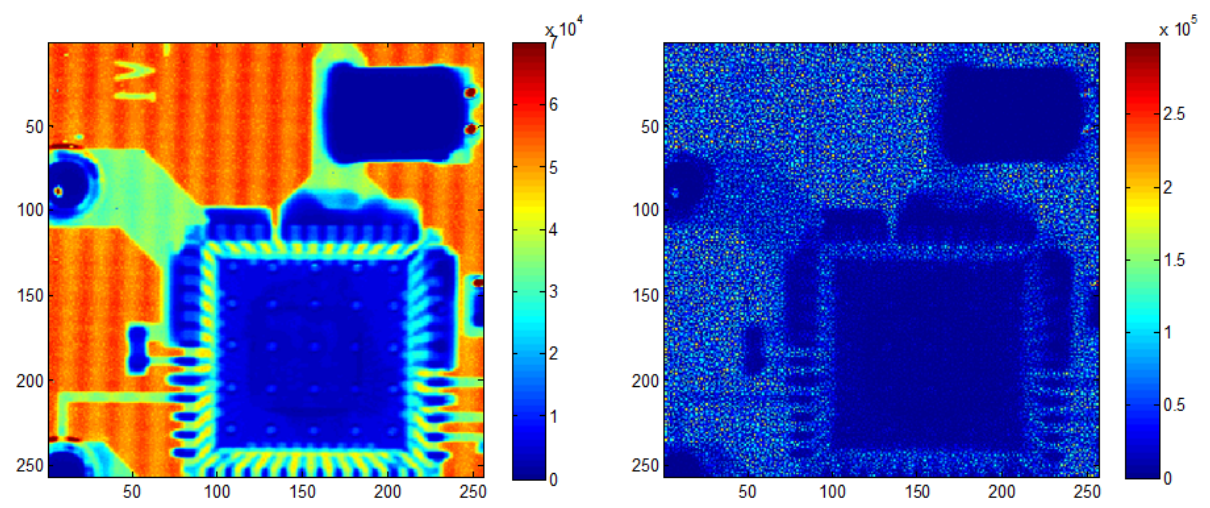

Figure 10. Comparison of two images of an electronic device in single pixel mode (left) and charge summing mode (right). The effect of the misallocation of hits is evident.

degradation in energy resolution due to charge sharing it is still possible to observe the influence of the threshold on the detected images the more dense parts becoming more transparent at the higher energy threshold.

A further single threshold image of a small leaf encapsulated in a tape was taken using a ${ }^{55} \mathrm{Fe}$ source with a very long exposure time of 12 hours and is shown in figure 7 . It is possible to distinguish the air bubbles around the leaf as well as some wrinkles in the tape near to the top of the image. Clearly all pixels are operating at a threshold below $5.9 \mathrm{keV}$.

Some further planar X-ray images have been taken in Freiburg to investigate the ability of the Medipix 3 for small animal imaging with high resolution. The chip was bump bonded to Si sensor material as well and the setup uses a microfocus X-ray source to enable imaging with up to $15 \mathrm{x}$ geometrical magnification. These images are taken using a scanned detector which provides a larger detection area. The target material of the tube was $\mathrm{W}$, the tube had a $150 \mu \mathrm{m}$ Be filter at the output and the tube voltage was $20 \mathrm{kV}$. The threshold was set to $6 \mathrm{keV}$ and the chip was 
used in the 24-Bit single pixel mode. A flat field correction and masked pixel interpolation were performed here.

Figure 8 shows the foot of a mouse paw as a biological high contrast object in 10x magnification. While the left image uses the full histogram range resulting in low contrast for bones, but the ability to see skin tissue and hairs, the histogram of the right image is cropped to 0.35 providing a high contrast for bones.

Figure 9 demonstrates a lesser stag beetle (Dorcus parallelipipedus) as a biological low contrast object. The high dynamic range of the Medipix 3 in combination with its almost noiseless function principle and its low threshold energy enables inner parts like the alimentary canal to be resolved through the chitin shell just as clearly as external parts like the eyes and the leg anchors.

The charge summing architecture guarantees that the energy of each photon is correctly reconstructed (figure 3) and assigned to a single pixel circuit even if the energy deposition takes place at the edges or corners of the pixels [5]. However, the unexpectedly high variation in the threshold from pixel to pixel even after equalization has an impact in the arbitration decision. Pixels with a lower threshold with respect to their neighbours will have a higher probability to get the hits assigned. This creates some granularity in the image. This is illustrated in figure 10. In the charge summing mode image histogram most of the counts are assigned to a small fraction of pixels i.e. those with a lower threshold in their neighbourhood. This issue will be solved in a future iteration of the chip with a modification in the allocation architecture.

\section{Summary and further work}

The first version of the Medipix 3 has been successfully bump bonded to high resistivity $300 \mu \mathrm{m}$ thick Si sensor. The initial electrical measurements have been confirmed using radioactive sources as calibration. The front-end noise is $\sim 75 \mathrm{e}$ rms in single pixel mode, $\sim 150 \mathrm{e} \mathrm{rms}$ in charge summing mode. The count rate has been measured in both modes indicating monotonic behaviour in single pixel mode and up to $17 \times 10^{6} \mathrm{ph} / \mathrm{mm} 2 / \mathrm{s}$ in charge summing mode. In single pixel mode the imaging quality of the device is excellent and the minimum threshold is $\sim 950 \mathrm{e} \mathrm{rms}$. The unexpectedly high pixeltopixel threshold variation in charge summing mode means that hit allocation is faulty and this needs to be improved in a subsequent version of the chip. First images using the chip connect to Si sensor material have been taken providing good high resolution results.

\section{Acknowledgments}

The authors are greatly indebted to the other members of the Medipix 3 Collaboration. In particular the groups lead by S. Pospisil at IEAP, Czech Technical University, Prague provided the readout system used for these measurements.

\section{References}

[1] X. Llopart et al., Medipix3: a 64k pixel detector readout chip working in single photon counting mode with improved spectrometric performance, in press in Nucl. Instrum. Meth. (corrected proof).

[2] R. Ballabriga et al., The Medipix 3 prototype, a pixel readout chip working in single photon counting mode with improved spectrometric performance, IEEE Trans. Nucl. Sci. 54 (2007) 1824. 
[3] R. Ballabriga, The design and implementation in $0.13 \mu \mathrm{m}$ CMOS of an algorithm permitting spectroscopic imaging with high spatial resolution for hybrid pixel detectors, $\mathrm{Ph} . \mathrm{D}$. thesis, Universitat Ramon Llull, Barcelona, Spain (2009), CERN-THESIS-2010-055.

[4] C. Schwarz, Digital X-ray imaging using hybrid semiconductor pixel detectors in single photon counting mode, Ph.D. Thesis, Albert-Ludwigs-Universität, Freiburg, Germany (2001).

[5] E.N. Gimenez et al., 3D Medipix2 detector characterization with a micro-focused X-ray beam, in pess in Nucl. Instrum. Meth. (corrected proof). 